\title{
Radiation therapy following targeted therapy in oligometastatic renal cell carcinoma
}

\author{
GWENAELLE GRAVIS ${ }^{1,2}$, MARJORIE FAURE ${ }^{1,3}$, STANISLAS RYBIKOWSKI $^{4}$, SLIMANE DERMECHE ${ }^{1}$, \\ MARGUERITE TYRAN ${ }^{2,5}$, BENOIT CALDERON $^{2,5}$, JEANNE THOMASSIN $^{6}$, JOCHEN WALZ $^{4}$ and NAJI SALEM ${ }^{5}$ \\ ${ }^{1}$ Department of Medical Oncology, Paoli-Calmettes Institute, 13009 Marseille; ${ }^{2}$ Research Unit Against Cancer, \\ CRCM-INSERM U1068, Faculty of Science of Luminy, Aix-Marseille University, 13009 Marseille; \\ ${ }^{3}$ Faculty of Medicine - Timone Sector, Aix-Marseille II University, 13385 Marseille; Departments of ${ }^{4}$ Urology, \\ ${ }^{5}$ Radiotherapy and ${ }^{6}$ Pathological Anatomy, Paoli-Calmettes Institute, 13009 Marseille, France
}

Received June 3, 2015; Accepted July 22, 2015

DOI: $10.3892 / \mathrm{mco} .2015 .613$

\begin{abstract}
Up to $40 \%$ of patients with renal cell carcinoma (RCC) with initially localized disease eventually develop metastasis following nephrectomy. The current standard of care for metastatic RCC (mRCC) is targeted therapy. However, complete response remains rare. A state of oligometastatic disease may exist, in which metastases are present in a limited number of locations; such cases may benefit from metastasis-directed local therapy, based on the evidence supporting resection of limited-volume metastases, allowing for improved disease control. We retrospectively analyzed 7 cases of response of RCC metastases, in patients treated with targeted therapies followed by radiation therapy (RT) of residual metastatic lesions in Paoli-Calmettes Institute (Marseille, France). We analyzed disease response rates, response to sequential strategy, relapse at the irradiated locations and disease evolution. The median follow-up was 34.1 months (range, 19.2-54.5 months). No progression at the irradiated sites was observed. A total of 5 patients had stable disease at the irradiated locations at the last follow-up; 3 remained in complete remission at the assessment, and 2 were stable. Excellent local response and clinical benefit may be achieved without added toxicity. In conclusion, sequential therapeutic strategies with RT following systemic treatment using sunitinib appear to be highly effective in patients with progressive $\mathrm{mRCC}$ and prompt the conduction of further confirmatory trials.
\end{abstract}

\section{Introduction}

One-third of patients diagnosed with renal cell carcinoma (RCC) present with metastasis at initial diagnosis

Correspondence to: Dr Gwenaelle Gravis, Department of Medical Oncology, Paoli-Calmettes Institute, 232 Sainte Marguerite Boulevard, 13009 Marseille, France

E-mail: gravisg@ipc.unicancer.fr

Key words: renal cell carcinoma, oligometastasis, targeted therapy, radiation therapy, sequential therapy and up to $40 \%$ of patients with initially localized RCC eventually develop metastasis following radical or partial nephrectomy (1). The current standard of care for patients with metastatic RCC (mRCC) is targeted therapy, which may reduce tumor burden and improve survival rates compared with historical treatments (2). However, complete response to systemic therapy alone remains extremely rare (3).

$\mathrm{mRCC}$ has long been a disease for which the resection of metastatic deposits has been considered for patient treatment (4). Patients who undergo metastasectomy tend to exhibit a better outcome, independently of their risk score (5).

However, in several cases, patients with metastatic disease are either unfit for surgery or have technically unresectable disease and, therefore, non-invasive metastasis-directed therapy for selected patients may be recommended.

For patients with metastatic disease progression and for those who present with distant disease, the median survival is 20 months, with an expected 5-year survival rate of 30\% (6).

Among patients with $\mathrm{mRCC}$, certain subgroups have been identified with improved prognosis and a prolonged disease course (7). For these patients, management of limited metastatic disease may be beneficial. A state of oligometastasis may exist, in which metastases are present in a limited number of locations and may benefit from metastasis-directed local therapy, based on the evidence supporting resection of limited-volume metastases. In addition to the oligometastatic state, a state of oligorecurrence has been hypothesized, whereby the primary site is controlled and the number and sites of metastasis are limited (8). However, locally treating all disease sites may be challenging.

The aim of the present retrospective study was to evaluate the role of adjuvant radiotherapy (RT) following response to an anti-angiogenic therapy on residual oligometastases.

\section{Patients and methods}

Patients. Between 2005 and 2011, 7 patients were treated in Paoli-Calmettes Institute (a comprehensive cancer center) for clear-cell mRCC in remission or with stable disease following tyrosine kinase inhibitor (TKI) treatment. In these patients, 
the metastatic sites were not considered resectable, but could receive local RT treatment. TKI was definitely discontinued prior to RT until disease progression.

Data regarding patient and tumor characteristics, metastatic presentation and clinical and biological prognostic factors were collected and reviewed.

The patients provided written informed consent, and the study was approved by the local Institutional Review Board of Paoli-Calmettes Institute.

Patient evaluation. The patients underwent computerized tomography of the chest, abdomen and pelvis following RT and every 3 months thereafter; they also underwent routine laboratory evaluations according to the systemic therapy schedule while on treatment.

\section{Results}

Patient characteristics. A total of 7 patients were enrolled in this study, including 2 women and 5 men. The median age was 66 years (range, 50.7-78.2 years). All the patients were diagnosed with clear-cell RCC. According to the Memorial Sloan-Kettering Cancer Center risk assessment tool, the risk was low for 2, intermediate for 3 and unknown for the remaining 2 patients.

The median time between RCC diagnosis and metastatic disease was 34.03 months (range, 20.16-110.16 months), and the time between the diagnosis of metastasis and RT was 35.3 months (range, 2.7-52.9 months). Overall, the patients received a minimum of one line of anti-angiogenic therapy (3 patients received $\geq 2$ lines).

All the patients were in partial remission or had stable disease at the end of the anti-angiogenic treatment and none were suitable for surgical removal of the metastases. Anti-angiogenic treatment was definitely discontinued prior to RT, until disease progression following RT. The irradiated metastatic sites included the mediastinal lymph nodes $(n=3)$, bones $(n=3)$, adrenal gland $(n=1)$ and pulmonary tissue $(n=1)$. One patient had bone and mediastinal lymph node metastases, which were treated simultaneously.

$R T$. The RT delivery technique was not homogeneous, similar to the totally delivered radiation dose. A total of 5 patients (3 with bone metastases and 3 with mediastinal lymph node metastases) received conformal RT with a planned dose of $36 \mathrm{~Gy}$ in 12 fractions, delivered to all 5 patients, but interrupted in 1 patient at 15 Gy due to symptomatic pleural effusion. Two patients, one with isolated lung metastasis and the second with adrenal gland metastasis, received extracranial stereotactic RT to a total dose of 42 Gy in 6 fractions and 40 Gy in 5 fractions, respectively. None of these patients developed unexpected or grade $>2$ RT-related toxicity.

At the end of the RT treatment, 2 patients had achieved a complete response of the irradiated site (lymph nodes), whereas the remaining 5 patients achieved partial response or stable disease.

Follow-up. The median follow-up time was 34.1 months (range, 19.2-54.5 months). No progression at the irradiated sites was observed during this time.
Two patients presented metastatic relapse on another site: 1 patient developed breast and pancreatic disease progression at 6 months following completion of bone RT, and was retreated by sunitinib, whereas the other patient developed a contralateral kidney nodule 13 months following completion of lymph node irradiation, and was treated by radiofrequency. The median time to relapse was 9.3 months (range, 7.5-12.6 months).

A total of 5 patients had stable disease at the irradiated locations at the last follow-up; 3 remained in complete remission. Of these patients, 1 died from a stroke. That patient had undergone RT of the metastatic disease 34.1 months prior. Since then, no systemic therapy had been reintroduced, and the patient exhibited favorable disease evolution at the assessment. The remaining 2 patients were considered as stable, with no relapse at the irradiated locations, without any further systemic therapy.

\section{Discussion}

We retrospectively analyzed the results of RT for metastatic sites non-suitable for surgery in patients with $\mathrm{mRCC}$ responsive to treatment following TKI administration.

The patients exhibited promising response rates and long-term response (median, $>24$ months) of the irradiated locations.

The majority of these patients exhibited a response to sequential therapies; 6 patients had received TKI prior to RT and 1 was administered sunitinib 9 months after completion of RT. A total of 5 patients had stable disease at the irradiated sites and 3 remained in complete remission. In terms of systemic disease, we observed that over half of the patients achieved complete response or stable disease (9).

$\mathrm{RCC}$ is considered to be radioresistant. Consequently, RT is mainly used for palliation of metastases or local tumor growth. The dogma regarding the radioresistance of RCC is based on the incorrect assumption that all RTs are equal. In 2014, a review by Daliani et al (10) summarized the emergence and important role of RT in the management of RCC and provided biological insights for the radiosensitivity of RCC. That study focused on the value of RT in the non-palliative treatment of extracranial disease and demonstrated how new advances in RT, such as stereotactic body RT (i.e., RT to an ablative dose) may affect treatment outcome.

As regards the radiobiological difference between high and low dose per fraction of ionizing radiation, it is known that the conventional dose of 1.8-3 Gy/fraction may not cause the necessary endothelial apoptotic response required for tumor death. By contrast, higher RT fractions efficiently destroy tumor vessels (microvasculature) (11) and are, therefore, expected to achieve better results in tumors that are highly dependent on angiogenesis. The majority of clear-cell RCCs are highly vascularized. Targeting of endothelial cells in RCC with RT is important, although it may be complicated.

The majority of evidence in favor of stereotactic body RT for RCC comes from single-institution studies (12). Thus far, there is no level 1 evidence, as no randomized trials have been performed.

The biological rationale supporting stereotactic body RT for mRCC also provides a basis for combined treatment 
with targeted drugs, such as sunitinib, which inhibits the molecular signaling of various receptor tyrosine kinases, including all receptors for platelet-derived growth factors and vascular endothelial growth factor (VEGF), mainly the VEGF receptor (13). Experimental data indicate that both VEGF and basic fibroblast growth factor inhibit radiation-induced endothelial apoptosis, thereby counteracting damages to the endothelium induced by single-fraction stereotactic body RT (14). Due to its inhibitory effects on VEGF, it is logical that sunitinib (among other TKIs) may act as a radiosensitizer for stereotactic body RT or single-fraction stereotactic body RT (15).

Over the past decade, research on RCC treatment has been focused on the development and implementation of targeted drugs (16), including TKIs, monoclonal antibodies and mammalian target of rapamycin inhibitors. These drugs have significantly changed the management of RCC and prolonged progression-free survival (17). However, these treatments are also associated with moderate toxicity (18).

With the widespread availability of modern RT techniques and online image guidance, there are no longer technical barriers to the application of stereotactic body RT for the treatment of oligometastatic RCC. Involvement of dedicated radiation oncologists in international congresses, advisory boards and scientific committees may also help to increase awareness of the value of stereotactic body RT in the treatment of RCC.

In conclusion, we investigated 7 cases of response of metastases to sequential therapy with targeted therapies followed by RT. To the best of our knowledge, this is the first description of response of metastatic sites to sequential therapy with sunitinib followed by RT for clear-cell mRCC. Sequential therapeutic strategies with RT following systemic sunitinib treatment appear to be highly effective in patients with progressive mRCC and prompt the conduction of confirmatory trials. Excellent local response and clinical benefits may be achieved without added toxicity. Sequential strategies with TKIs followed by RT are promising, although they require further investigation, since, thus far, all available studies investigated the concomitant administration of these therapies.

\section{Acknowledgements}

We would like to thank Elise Coessens for her assistance with the preparation of this manuscript.

\section{References}

1. Escudier B: Advanced renal cell carcinoma: Current and emerging management strategies. Drugs 67: 1257-1264, 2007.

2. Wuthrick EJ, Kamrava M, Curran WJ Jr, Werner-Wasik M, Camphausen KA, Hyslop T, Axelrod R, Andrews DW, Glass J, Machtay M, et al: A phase $1 \mathrm{~b}$ trial of the combination of the antiangiogenic agent sunitinib and radiation therapy for patients with primary and metastatic central nervous system malignancies. Cancer 117: 5548-5559, 2011.
3. Albiges L, Oudard S, Negrier S, Caty A, Gravis G, Joly F, Duclos B, Geoffrois L, Rolland F, Guillot A, et al: Complete remission with tyrosine kinase inhibitors in renal cell carcinoma. J Clin Oncol 30: 482-487, 2012.

4. Zhang HP, Takayama K, Su B, Jiao XD, Li R and Wang JJ: Effect of sunitinib combined with ionizing radiation on endothelial cells. J Radiat Res (Tokyo) 52: 1-8, 2011.

5. Walsh L, Stanfield JL, Cho LC, Chang CH, Forster K, Kabbani W, Cadeddu JA, Hsieh JT, Choy H, Timmerman R, et al: Efficacy of ablative high-dose-per-fraction radiation for implanted human renal cell cancer in a nude mouse model. Eur Urol 50: 795-800, discussion 800, 2006.

6. Pellerin O, Medioni J, Vulser C, Déan C, Oudard S and Sapoval M: Management of painful pelvic bone metastasis of renal cell carcinoma using embolization, radio-frequency ablation, and cementoplasty: A prospective evaluation of efficacy and safety. Cardiovasc Intervent Radiol 37: 730-736, 2014.

7. Niibe $\mathrm{Y}$ and Hayakawa K: Oligometastases and oligo-recurrence: The new era of cancer therapy. Jpn J Clin Oncol 40: 107-111, 2010.

8. Motzer RJ, Hutson TE, Tomczak P, Michaelson MD, Bukowski RM, Oudard S, Negrier S, Szczylik C, Pili R, Bjarnason GA, et al: Overall survival and updated results for sunitinib compared with interferon alfa in patients with metastatic renal cell carcinoma. J Clin Oncol 27: 3584-3590, 2009.

9. Tsui KH, Shvarts O, Smith RB, Figlin RA, deKernion JB and Belldegrun A: Prognostic indicators for renal cell carcinoma: A multivariate analysis of 643 patients using the revised 1997 TNM staging criteria. J Urol 163: 1090-1095, quiz 1295, 2000.

10. Daliani DD, Tannir NM, Papandreou CN, Wang X, Swisher S, Wood CG, Swanson DA, Logothetis CJ and Jonasch E: Prospective assessment of systemic therapy followed by surgical removal of metastases in selected patients with renal cell carcinoma. BJU Int 104: 456-460, 2009.

11. De Meerleer G, Khoo V, Escudier B, Joniau S, Bossi A, Ost P, Briganti A, Fonteyne V, Van Vulpen M, Lumen N, et al: Radiotherapy for renal-cell carcinoma. Lancet Oncol 15: e170-e177, 2014

12. Escudier B and Kataja V; ESMO Guidelines Working Group: Renal cell carcinoma: ESMO Clinical Practice Guidelines for diagnosis, treatment and follow-up. Ann Oncol 21 (Suppl 5): v137-v139, 2010.

13. Escudier B, Eisen T, Stadler WM, Szczylik C, Oudard S, Siebels M, Negrier S, Chevreau C, Solska E, Desai AA, et al; TARGET Study Group: Sorafenib in advanced clear-cell renal-cell carcinoma. N Engl J Med 356: 125-134, 2007.

14. Stinauer MA, Kavanagh BD, Schefter TE, Gonzalez R, Flaig T, Lewis K, Robinson W, Chidel M, Glode M and Raben D: Stereotactic body radiation therapy for melanoma and renal cell carcinoma: Impact of single fraction equivalent dose on local control. Radiat Oncol 6: 34, 2011.

15. Motzer RJ, Mazumdar M, Bacik J, Berg W, Amsterdam A and Ferrara J: Survival and prognostic stratification of 670 patients with advanced renal cell carcinoma. J Clin Oncol 17: 2530-2540, 1999.

16. Russo P, Jang TL, Pettus JA, Huang WC, Eggener SE, O'Brien MF, Karellas ME, Karanikolas NT and Kagiwada MA: Survival rates after resection for localized kidney cancer: 1989 to 2004. Cancer 113: 84-96, 2008.

17. Pantuck AJ, Zisman A and Belldegrun AS: The changing natural history of renal cell carcinoma. J Urol 166: 1611-1623, 2001.

18. Zelefsky MJ, Greco C, Motzer R, Magsanoc JM, Pei X, Lovelock M, Mechalakos J, Zatcky J, Fuks Z and Yamada Y: Tumor control outcomes after hypofractionated and single-dose stereotactic image-guided intensity-modulated radiotherapy for extracranial metastases from renal cell carcinoma. Int J Radiat Oncol Biol Phys 82: 1744-1748, 2012. 